\title{
BMJ Open Impact of a Pharmacist-included Mobile Geriatrics team intervention on potentially inappropriate drug prescribing: protocol for a prospective feasibility study (PharMoG study)
}

To cite: Pagès $A$, Roland $C$, Qassemi S, et al. Impact of a Pharmacist-included Mobile Geriatrics team intervention on potentially inappropriate drug prescribing: protocol for a prospective feasibility study (PharMoG study). BMJ Open 2020;10:e040917. doi:10.1136/ bmjopen-2020-040917

- Prepublication history and additional material for this paper is available online. To view these files, please visit the journal online (http://dx.doi.org/10. 1136/bmjopen-2020-040917)

Received 25 May 2020 Revised 02 November 2020 Accepted 02 November 2020

Check for updates

(c) Author(s) (or their employer(s)) 2020. Re-use permitted under CC BY-NC. No commercial re-use. See rights and permissions. Published by BMJ.

For numbered affiliations see end of article.

Correspondence to

Dr Arnaud Pagès;

pages.ar@chu-toulouse.fr

\section{ABSTRACT}

Introduction Research has shown that potentially inappropriate drug prescription (PIDP) is highly prevalent in older people. The presence of PIDPs is associated with adverse health outcomes. This study aims to evaluate the impact of a PHARmacist-included MObile Geriatrics (PharMoG) team intervention on PIDPs in older patients hospitalised in the medical, surgical and emergency departments of a university hospital.

Methods and analysis The PharMoG study is a prospective, interventional, single-centre feasibility study describing the impact of a PharMoG team on PIDPs in older hospitalised patients. Pharmacist intervention will be a treatment optimisation (clinical medication review) based on a combination of explicit and implicit criteria to detect PIDPs. The primary outcome is the acceptance rate of the mobile team's proposed treatment optimisations related to PIDPs, measured at the patient's discharge from the department. This pharmacist will work in cooperation with the physician of the mobile geriatric team. After the intervention of the mobile geriatric team, the proposals for improving therapy will be sent to the hospital medical team caring for the patient and to the patient's attending physician. The patient will be followed for 3 months after discharge from the hospital.

Ethics and dissemination This study was approved by the South-West and Overseas Territories II Ethics Committee. Oral consent must be obtained prior to participation, either from the patient or from the patient's representative (trusted person and/or a family member). The results will be presented at national and international conferences and published in peer-reviewed journals. Trial registration number NCT04151797.

\section{INTRODUCTION AND BACKGROUND}

In patients aged $\geq 75$ years, comorbidities are common ${ }^{1}$ and a cause of polypharmacy. Multiple prescriptions, combined with physiological changes in pharmacokinetic and pharmacodynamic parameters that occur
Strengths and limitations of this study

- This prospective study is the first to describe the intervention of a mobile geriatric team including a pharmacist in France.

- The pharmacist intervention consists of a clinical medication review based on both implicit and explicit criteria, which reproduces current practices.

- The acceptance rate of the mobile geriatric team propositions related to potentially inappropriate drug prescriptions is measured both at discharge and after 3 months to assess the long-term effects of the intervention.

- Our feasibility study is not randomised, with no control group.

- The detection of potentially inappropriate drug prescriptions is carried out by the pharmacist involved in the study and not by an external assessor (clinical pharmacist not involved in the study or computer algorithm)

with age, ${ }^{2}$ make older adults more susceptible to adverse drug reactions. Each new drug prescribed is thought to increase the rate of adverse effects by $12 \%-28 \%^{3}$ and the risk of hospitalisation by $11 \%{ }^{4}$

The term 'potentially inappropriate drug prescription' (PIDP) $)^{5}$ refers to

- overuse (use of prescription drugs that are not indicated or whose efficacy has not been demonstrated),

- misuse (use of drugs whose risks exceed the expected benefits),

- underuse (failure to use effective drugs in patients with conditions for which one or more drug classes have been proven effective). 
Several tools have been developed to make it easier to identify PIDPs using an explicit or implicit approach, or a combination of both. The implicit approach is based on clinical judgement: the risk/benefit ratio of each drug is analysed in light of the patient's history, concomitant illnesses, laboratory tests and coprescribed drugs (eg, the Medication Appropriateness Index (MAI)). The explicit approach is based on criteria generally determined by expert consensus. They consist of standardised lists of drugs to be avoided in older subjects (eg, the European list of potentially inappropriate medications for older people (EU(7)-PIM list) $)^{6}$ or more complex rules combining drugs and clinical parameters (eg, STOPP/ START (Screening Tool of Older Persons' Prescriptions/ Screening Tool to Alert to Right Treatment) criteria). ${ }^{7}$

Many studies on the prevalence of PIDPs, their health impact and interventions to reduce them have been published. The prevalence of PIDPs varies considerably depending on the context and tools used to detect them. ${ }^{8}$ The Gallagher et at study conducted in six European university hospitals evaluated the prevalence of PIDPs in older patients admitted for acute care to be $59.4 \%$ using the START criteria, $51.3 \%$ using the STOPP criteria and $30.4 \%$ using the Beers criteria. ${ }^{10}$ In our facility, a crosssectional, descriptive, observational study performed on outpatients in 2015 showed $71.2 \%$ of the 229 subjects to have a PIDP. ${ }^{11}$ Concerning the health impacts of PIDPs, a recent meta-analysis showed evidence of a connection between PIDPs and the risk of adverse effects and hospitalisations in older subjects. ${ }^{12}$ Finally, concerning strategies for avoiding PIDPs, a Cochrane literature review recently established that the presence of a pharmacist, especially as part of a multidisciplinary team, reduces PIDPs. ${ }^{13}$

Regarding the role of pharmacists, several studies have shown that a pharmaceutical analysis of prescriptions and treatment optimisation has a positive impact on reducing adverse effects, length of hospitalisation, readmission rate, quality of life and mortality. ${ }^{514-16}$ The impact of a multidisciplinary intervention involving nurses, pharmacists and physicians is also well established in the hospital, ${ }^{17}$ especially on PIDPs and adverse events linked to drug therapy. ${ }^{18-21}$ The impact of this type of multidisciplinary approach has rarely been evaluated in France. ${ }^{22} 23$

Among the multidisciplinary teams intervening in health facilities are mobile geriatric teams (MGTs). There are more than 200 MGTs in France. They intervene in non-geriatric medical, surgical and emergency department services to provide geriatric evaluation and advice through a multidisciplinary evaluation. In 2013, a metaanalysis showed that MGTs have a positive impact on the mortality rate at 6 months (relative risk (RR): 0.66 ; 95\% CI: 0.52 to 0.85 ) and 8 months (RR: $0.51 ; 95 \% \mathrm{CI}$ : 0.31 to 0.85 ) after hospital discharge. ${ }^{24}$ We have found no published studies evaluating the impact of MGTs on drug prescriptions specifically, particularly inappropriate ones. However, the characteristics of patients seen by MGTs match those most at risk of adverse drug reactions and PIDPs. ${ }^{25}$ In other countries, geriatric consultation teams that are multidisciplinary but do not include pharmacists have helped reduce PIDPs both for inpatients ${ }^{26}$ and for hospitalised patients. ${ }^{27}$

In summary, the published data show the following:

- The relationship between PIDPs, frequent adverse effects and risk of hospitalisation.

- The impact of MGTs on the mortality rate, 6 and 8 months after hospital discharge.

- The impact of clinical pharmacy activities and multidisciplinary treatment optimisation on PIDPs, length of hospitalisation and the rate of unscheduled readmissions.

- The relationship between iatrogenic risk and a lack of coordination between professionals.

There are currently no data, either French or international, on the following:

- The impact of MGTs on PIDPs in France.

- The impact of including a pharmacist in the MGT (ie, a PHARmacist-included MObile Geriatrics (PharMoG) team).

We hypothesise that a PharMoG team intervention including a clinical medication review improves the quality, safety and relevance of drug treatment in older patients by decreasing exposure to potentially inappropriate drugs and improving cooperation between pharmacists and doctors caring for older patients.

\section{METHODS AND ANALYSIS \\ Design}

The PharMoG study is a prospective, interventional, single-centre feasibility study describing the intervention of a PharMoG team on PIDPs in older patients hospitalised at Toulouse University Hospital, France. The checklist items from the 'Standard Protocol Items Recommendations for Interventional Trials' and the 'Consolidated Standards of Reporting Trials extension for the reporting of randomised pilot and feasibility studies' (only items regarding feasibility studies) were used to report this study protocol. ${ }^{28} 29$

\section{Patients}

\section{Consent and inclusion}

The MGT intervenes only at the request of the patient's hospitalist for a geriatric opinion. The request is made by telephone or through the hospital's software.

During the screening visit, if the inclusion criteria are met, the investigator (geriatrician or pharmacist) gives the subject or the subject's representative (trusted person or family member) a copy of the information sheet and answers any questions about the purpose, constraints, foreseeable risks and expected benefits of the study. The investigator also specifies the subject's rights in a research study and verifies the eligibility criteria. After this information session, the subject or the subject's representative is given time ( 1 day) to think it over. If the patient agrees to participate in the study, he or she consents orally as recommended by French law (French Law No. 2012-300 
of 5 March 2012 on human research trials (the Jardé Act)). Oral consent is sufficient because according to French law (the Jardé Act), this is a minimal-risk research project. ${ }^{30}$ If the patient does not have the cognitive or physical capacity to read the information sheet, the investigator will address the patient's trusted person and/or family member, who will consent orally on behalf of the patient. A copy of the information sheet and consent form for the patient or the patient's trusted person and/ or family member (translated into English) is provided as online supplemental file 1 . The investigator will record the representative's consent in the patient's medical file. The investigator assigns an enrolment number to the subject and keeps an up-to-date key (with the name and enrolment number), separate from the electronic case report form (e-CRF).

\section{Sample size and duration}

As this is a feasibility study to describe the intervention of a PharMoG team and to obtain preliminary results and feasibility outcomes, we did not calculate a sample size based on assumed efficacy. The number of patients and the duration of the study are given for information only and have been estimated to have a sufficient sample of patients to evaluate feasibility.

The enrolment period is expected to allow sufficient time to gather data on approximately 250 patients, ensuring that the prescriptions analysed and clinical situations are representative in the context of a descriptive study. Knowing that the MGT of Toulouse University Hospital treats approximately 1000 patients per year, we can estimate that it would take 1 year to enrol 250 patients, taking into account the inclusion criteria and availability of the pharmacist in the MGT. Knowing that each patient is followed for 3 months after the PharMoG intervention, we can estimate that the whole study would take around 15 months, assuming a maximum hospital stay of 2 months. We chose a 3-month follow-up because it would be consistent with a new general practitioner visit and also because this duration was used in another study on the effects of a clinical medication review. ${ }^{31}$

\section{Inclusion criteria}

Participants with the following criteria will be included in the study:

- Man or woman aged $\geq 75$ years.

- Hospitalised at Toulouse University Hospital in a medical, surgical or emergency department, with admissions having requested the MGT.

- Having five or more prescription drugs before the intervention (including all routes of administration and as-needed prescriptions).

- Having given oral consent to participate in the study (or oral consent given by the representative: a trusted person and/or a family member of the patient, if necessary).

- Belonging to a social security scheme or equivalent.
Exclusion criteria

Participants with the following criteria will be excluded from the study:

- Man or woman $<75$ years of age.

- Not hospitalised in any of the departments targeted by the study (medicine, surgery, emergency).

- Not having had the MGT called.

- Having fewer than five prescription drugs before the intervention (including all routes of administration and as-needed prescriptions).

- Legally protected adults (under guardianship or protection of the court).

- Patient participating in another research protocol.

\section{Intervention}

All the pharmacists in the PharMoG team have specific training in clinical pharmacy and pharmacology applied to geriatrics achieved through a university diploma programme ('Optimization of drug management of the elderly patient'). The pharmacists of the PharMoG team review the patient's medical file to look for the following information: medical and surgical history, comorbidities, the reason for hospitalisation, the reason the PharMoG team was called, medicines prescribed, and information important to analysing the prescription and left to the pharmacist's discretion (falls, malnutrition, insomnia, urinary incontinence, frailty or dependence, adverse effects, laboratory results, etc). As the usual care provided by pharmacists is not uniform at our hospital (pharmaceutical analysis, medication review, medication reconciliation, pharmaceutical interview), the pharmacist in the PharMoG team contacts the department's pharmaceutical team and collects information on the actions already carried out.

Based on the information gathered through a routine medication reconciliation, the study pharmacist conducts a clinical medication review as recommended by the French Society of Clinical Pharmacy ${ }^{32}$ based on both explicit and implicit approaches. As there are many explicit criteria tools available, we chose the EU(7)-PIM list ${ }^{6}$ and the STOPP and START V.2 criteria ${ }^{7}$ because they were the most recent tools in Europe, they were validated for both inpatients and outpatients, and the combination of the two allowed us to detect situations of overuse, misuse and underuse. ${ }^{33}$ They were supplemented by the French Alert and Mastering of drug Iatrogenicity (AMI) indicators, 'medical benefit' assessed by the FrenchNational Authority for Health (HAS) and Summary of Product Characteristics of the drugs. For the implicit approach, we took into account the patient's comorbidities, laboratory test results, adverse events reported and questions from the MAI. ${ }^{34}$ Appropriateness or inappropriateness is assessed by calculating the number of PIDPs for each drug prescription line.

The study pharmacist discusses the proposed pharmaceutical inventions with the MGT and then writes them up. The geriatrician adds these proposals to the computerised report and sends it to the hospital physician in 
charge of the patient and the attending physician, and then to the community pharmacist by secured electronic messaging. The PharMoG team's therapeutic optimisation proposals are transmitted to the community pharmacist to relay the pharmaceutical interventions carried out by the team and to reinforce them by carrying out a postdischarge clinical medication review in primary care.

\section{End points}

Primary end point

The primary end point is the acceptance rate of the PharMoG team's proposed treatment optimisations related to PIDPs, measured at patient's discharge from the department.

\section{Secondary end points}

The secondary end points are the following:

- The change in the average number of PIDPs per patient, before the intervention, at the time of discharge and 3 months after the intervention by the mobile team.

- The percentage of PIDPs per patient, before the intervention, at the time of discharge and 3 months after the intervention by the mobile team.

- The percentage of patients with at least one PIDP before the intervention, at the time of discharge or 3 months after the intervention by the mobile team.

- The number of prescription medicines per patient, before the intervention, at the time of discharge and 3 months after the intervention by the mobile team.

- The acceptance rate of the mobile team's proposed treatment optimisations on the entire drug prescription, at the time of discharge and 3 months after the intervention by the mobile team.

- The number of postdischarge clinical medication reviews performed by community pharmacists.

- Falls within 3 months after the mobile team's intervention.

- Mortality 3 months after the mobile team's intervention.

- Hospitalisation, emergency department visitswhether or not admitted to the hospital-and institutionalisation within 3 months after the mobile team's intervention.

- Changes in the cost per patient of medications prescribed before the intervention, at the time of discharge and 3 months after the PharMoG team's intervention, according to the rates reimbursable by national health insurance, or failing that, according to the price of hospital purchases.

The end points will be assessed 3 months after the mobile team's intervention only for patients whose hospital stay does not exceed 2 months after the mobile team's intervention.

The other end points for judging the feasibility of the study and satisfaction of health professionals are the following:

- The number of enrolments per day.
- The time it takes for treatment optimisation of drug prescriptions (calculated as the difference between the time the pharmacist arrives and the time the pharmacist leaves the ward).

- The proportion of patients enrolled/the number of patients meeting the inclusion criteria.

- The number of patients lost to follow-up 3 months after the mobile team's intervention.

- Satisfaction of the physicians in the PharMoG team and of the departments involved according to the Likert scale (satisfaction questionnaire will be sent at the end of the study).

\section{Data collection}

The data collection methods are detailed in table 1 . The data are collected in a CRF and then in an e-CRF.

Data will be collected based on the patient's computerised record, the patient's paper record and if, appropriate, an interview with the patient or the trusted person and/or family member of the patient. If the department in which the patient is hospitalised normally has a pharmaceutical team, the study pharmacist will contact them to inform them that the patient has been enrolled and to gather information about what has already been done (medication reconciliations on admission, previous pharmaceutical interventions). In addition, if necessary, the investigator will contact the community pharmacist to find out which treatments are usually taken at home by the patient.

For the callback at the time of discharge, the data will be collected by the pharmacist of the mobile team or the clinical research associate by telephone, and/or by a visit to the department in question, and/or from data in the patient's computerised file. For the 3-month callback $( \pm 15$ days) after discharge from the hospital, the data are collected by the pharmacist of the MGT or the clinical research associate by a telephone call to the patient (and/ or the patient's trusted person and/or family member, if necessary) and from the community pharmacist. The following data will be collected: drugs prescribed, falls, hospitalisations, emergency department visits and institutionalisation. A fall is defined as 'an event which results in a person coming to rest inadvertently on the ground or floor or other lower level' by WHO. ${ }^{35}$ The occurrence of falls is collected by consulting different sources: patient records and interviews with the patient, family and general practitioner.

A participant may stop participating in the study at any time without any consequences for him or her or for his or her subsequent care. If withdrawal from the study occurs before hospitalisation (death, withdrawal of consent, etc), the main end point is not calculated. In the event of withdrawal from the study, there is no provision for replacement of participants.

\section{Data analysis}

Concerning the statistics regarding the primary and secondary end points: 


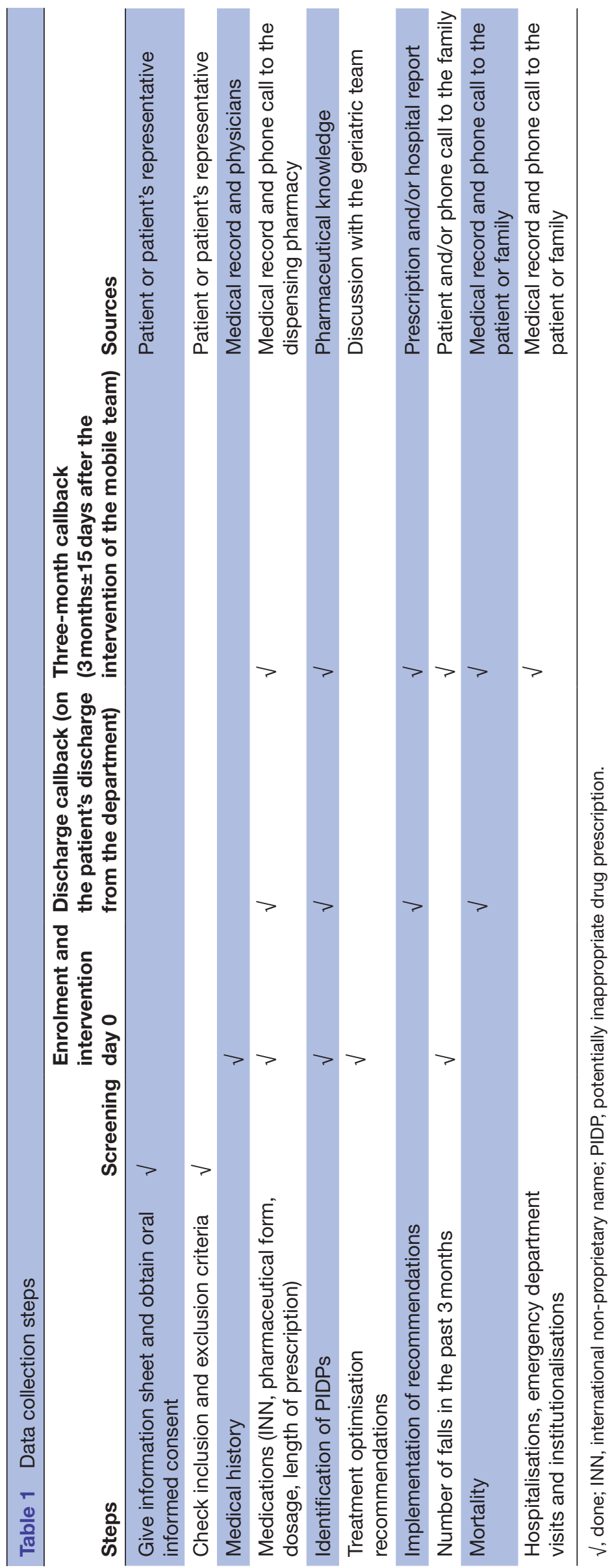


- The quantitative variables are described in terms of the mean and SD or as a range and compared using Student's t-test for paired data if the distributions are normal and a Wilcoxon signed-rank test if they are not.

- The qualitative variables will be described as numbers and percentages and compared using a McNemar test if the validity conditions are met and a Fisher exact test if they are not.

- For economic data, the results will be presented in the form of incremental costs per patient and 95\% CIs from a bootstrap. The economic analysis will be done from a national health insurance point of view using a time horizon of 1 month.

The significance threshold will be set at 0.05 , and all the tests will be two-tailed.

A mixed model will be used to explain the acceptance rate of the PharMoG team's proposed treatment optimisations and the changes in PIDPs before and after the intervention of the PharMoG team by the following:

- The patients' characteristics: age, gender, weight, number of medicines before the intervention, comorbidity index (Charlson) ${ }^{36}$ and adverse drug event risk score in geriatrics (Trivalle score ${ }^{37}$ ).

- The type of department (surgical vs medicine vs emergency, and the presence and level of pharmaceutical analysis according to the French Society of Clinical Pharmacology).

SAS software V.9.3 (SAS Institute) will be used to produce the statistical outcomes.

\section{Patient and public involvement}

It was not possible to involve patients or the public in the design, or conduct, or reporting, or dissemination plans of our research.

\section{DISCUSSION}

This feasibility study aims to obtain preliminary results on the impact of a PharMoG on PIDPs in older, hospitalised patients and to ensure the feasibility of this type of intervention.

First, regarding the main end point, we chose to detect PIDPs using a method combining several explicit criteria and an implicit approach. On one hand, this choice is consistent with current practice and provides patients with pharmaceutical care that is as personalised as possible. On the other hand, this approach is less reproducible than the application of explicit criteria from a single tool, even if all the pharmacists involved in the PharMoG team were provided with clinical pharmacy training applied to geriatrics. It should be emphasised that in this feasibility study, the PIDPs are evaluated by the pharmacist included in the MGT. To overcome this problem, in the future we could compare the number of PIDPs related to each drug prescription either via a computerised algorithm only for explicit criteria or by another clinical pharmacist.
Finally, as there is no control group, the results of this study cannot be used to assess the pharmacist's own added value within the MGT. We can hypothesise that the main added value of having a pharmacist on the PharMoG team compared with usual care therefore lies in the clinical medication review. To validate this hypothesis, if the results of this feasibility study are conclusive, we can consider launching a multicentre randomised study to demonstrate the efficacy and cost-effectiveness of this approach for older patients. To ensure the consistency of the intervention, we will provide clinical pharmacy training in geriatrics to all pharmacists involved in the study as well as a detailed description of the intervention (gathering information with a medication reconciliation and carrying out a clinical medication review with the tools to be used). The study design would be a comparison of two groups with cluster randomisation by medical wards to avoid contamination bias: a group of wards with MGT intervention without a pharmacist and a group of medical wards with a pharmacist-included MGT (PharMoG team).

\section{ETHICS AND DISSEMINATION}

The sponsor and investigator(s) agree to conduct this study in compliance with French Law No. 2012-300 of 5 March 2012 on human research trials (the Jardé Act), as well as with Good Clinical Practice (ICH version 4 of 9 November 2016 and the decision dated 24 November 2006) and the Helsinki Declaration. The study is conducted in accordance with this protocol. Other than in emergency situations requiring the use of specific therapeutic procedures, the investigator(s) agree to abide by the protocol in its entirety, particularly with regard to obtaining consent, and the notification and follow-up of serious adverse events. In this study, adverse events will have to be declared according to various health vigilance procedures (pharmacovigilance, medical device vigilance, haemovigilance) in accordance with the regulations in force. This study was approved by the South-West and Overseas Territories II Ethics Committee (2-19-081 id5236). The Toulouse University Hospital, the sponsor of this study, took out a liability insurance policy in accordance with French public health code provisions. An audit will be scheduled before including the 50 th patient. The next audit will be carried out at the end of the study. The article is based on version 2.2 of the protocol dated 16 December 2019. The study started in December 2019

The data recorded at the time of this study are processed in a computer at Toulouse University Hospital in accordance with French Law No. 78-17 of 6 January 1978 amended by Law No. 2018-493 of 20 June 2018 on Data Processing, Data Files and Individual Liberties (the French Data Protection Act) and Regulation No. 2016/679 adopted by the European Parliament on 16 April 2016, the General Data Protection Regulation. This study is governed by Reference Methodology (MR-001) under the provisions of Article 54, paragraph 5, of the 
French Data Protection Act. This change was ratified in a decision dated 5 January 2006 and updated on 21 July 2016. Toulouse University Hospital has signed an agreement to comply with this 'Reference Methodology'. The results will be presented at national and international conferences and published in peer-reviewed journals. This study is registered in the European Union Clinical Trials Register (IDRCB 2018-A00180-55) and at clinicaltrials.gov.

\section{Author affiliations}

${ }^{1}$ Department of Pharmacy, Toulouse University Hospital, Toulouse, France ${ }^{2}$ Institute of Aging, Gérontopôle, INSPIRE project, Toulouse University Hospital, Toulouse, France

${ }^{3}$ Centre for Epidemiology and Population Health Research (CERPOP), UMR 1027, Inserm, University of Toulouse (UPS), Toulouse, France

${ }^{4}$ Department of Geriatrics, Toulouse University Hospital, Toulouse, France

${ }^{5}$ Department of Research and Innovation, Toulouse University Hospital, Toulouse, France

\section{Twitter Arnaud Pagès @arndpgs}

Collaborators Members of PharMoG study group: principal investigator: Philippe Cestac; co-investigator: Thierry Voisin; coordination: Cécile McCambridge; clinical research associates: Audrey Belloc, Charlotte Dunet; investigators: Soraya Qassemi, Christel Roland; methodology: Sandrine Andrieu, Adeline Gallini; statistical analysis and data management: Blandine Juillard-Condat, Arnaud Pagès; Mobile geriatric team—supervisor: Thierry Voisin; medical doctors: Anne-Bahia Abdeljalil, Mathieu Houles, Marjolaine Romain, Oliver Toulza; nurses: Jessica Barbe, Lisa Bonometti; pharmacists: Soraya Qassemi, Christel Roland; secretary: Nadia Beldjebel.

Contributors All authors made substantial contributions to the conception or design of the protocol and approved the final version of the protocol. Specifically, $A B A, C M C, M H, M R, O T, P C$ and TV were involved in the conception of the protocol. $A P$ and $B J-C$ led the overall writing of the protocol and designed the database. SQ and $C R$ reviewed the protocol and validated the methods of data collection. $A B$ contributed to the ethical and regulatory aspects of the research. The PHARmacistincluded MObile Geriatrics (PharMoG) study group represents all persons involved in the implementation of the PharMoG study.

Funding The study is funded by the Toulouse University Hospital (ARI 2017— Local Research Program; Grant Number: RC31/170353).

Competing interests None declared.

Patient consent for publication Not required.

Provenance and peer review Not commissioned; externally peer reviewed.

Supplemental material This content has been supplied by the author(s). It has not been vetted by BMJ Publishing Group Limited (BMJ) and may not have been peer-reviewed. Any opinions or recommendations discussed are solely those of the author(s) and are not endorsed by BMJ. BMJ disclaims all liability and responsibility arising from any reliance placed on the content. Where the content includes any translated material, BMJ does not warrant the accuracy and reliability of the translations (including but not limited to local regulations, clinical guidelines, terminology, drug names and drug dosages), and is not responsible for any error and/or omissions arising from translation and adaptation or otherwise.

Open access This is an open access article distributed in accordance with the Creative Commons Attribution Non Commercial (CC BY-NC 4.0) license, which permits others to distribute, remix, adapt, build upon this work non-commercially, and license their derivative works on different terms, provided the original work is properly cited, appropriate credit is given, any changes made indicated, and the use is non-commercial. See: http://creativecommons.org/licenses/by-nc/4.0/.

ORCID iD

Arnaud Pagès http://orcid.org/0000-0002-2337-8693

\section{REFERENCES}

1 Salive ME. Multimorbidity in older adults. Epidemiol Rev 2013;35:75-83.
2 Mangoni AA, Jackson SHD. Age-Related changes in pharmacokinetics and pharmacodynamics: basic principles and practical applications. Br J Clin Pharmacol 2004;57:6-14.

3 Calderón-Larrañaga A, Poblador-Plou B, González-Rubio F, et al. Multimorbidity, polypharmacy, referrals, and adverse drug events: are we doing things well? Br J Gen Pract 2012;62:e821-6.

4 Wauters M, Elseviers M, Vaes B, et al. Mortality, hospitalisation, institutionalisation in community-dwelling oldest old: the impact of medication. Arch Gerontol Geriatr 2016;65:9-16.

5 Spinewine A, Schmader KE, Barber N, et al. Appropriate prescribing in elderly people: how well can it be measured and optimised? Lancet 2007;370:173-84.

6 Renom-Guiteras A, Meyer G, Thürmann PA. The EU(7)-PIM list: a list of potentially inappropriate medications for older people consented by experts from seven European countries. Eur J Clin Pharmacol 2015;71:861-75.

7 O'Mahony D, O'Sullivan D, Byrne S, et al. STOPP/START criteria for potentially inappropriate prescribing in older people: version 2. Age Ageing 2015;44:213-8.

8 Tommelein E, Mehuys E, Petrovic M, et al. Potentially inappropriate prescribing in community-dwelling older people across Europe: a systematic literature review. Eur J Clin Pharmacol 2015;71:1415-27.

9 Gallagher P, Ryan C, Byrne S, et al. STOPP (screening tool of older person's prescriptions) and start (screening tool to alert doctors to right treatment). consensus validation. Int $J$ Clin Pharmacol Ther 2008;46:72-83.

10 Beers MH. Explicit criteria for determining potentially inappropriate medication use by the elderly. An update. Arch Intern Med 1997;157:1531-6.

11 Récoché I, Lebaudy C, Cool C, et al. Potentially inappropriate prescribing in a population of frail elderly people. Int J Clin Pharm 2017;39:113-9.

12 Xing XX, Zhu C, Liang HY, et al. Associations between potentially inappropriate medications and adverse health outcomes in the elderly: a systematic review and meta-analysis. Ann Pharmacother 2019;53:1005-19.

13 Patterson SM, Cadogan CA, Kerse N, et al. Interventions to improve the appropriate use of polypharmacy for older people. Cochrane Database Syst Rev 2014;10:CD008165.

14 Simonson W, Feinberg JL. Medication-related problems in the elderly: defining the issues and identifying solutions. Drugs Aging 2005;22:559-69.

15 Alldred DP, Kennedy M-C, Hughes C, et al. Interventions to optimise prescribing for older people in care homes. Cochrane Database Syst Rev 2016;2:CD009095.

16 Patterson SM, Hughes C, Kerse N, et al. Interventions to improve the appropriate use of polypharmacy for older people. Cochrane Database Syst Rev 2012;5:CD008165.

17 Kaboli PJ, Hoth AB, McClimon BJ, et al. Clinical pharmacists and inpatient medical care: a systematic review. Arch Intern Med 2006;166:955-64.

18 Roberts MS, Stokes JA, King MA, et al. Outcomes of a randomized controlled trial of a clinical pharmacy intervention in 52 nursing homes. Br J Clin Pharmacol 2001;51:257-65.

19 Crotty M, Halbert J, Rowett D, et al. An outreach geriatric medication advisory service in residential aged care: a randomised controlled trial of case conferencing. Age Ageing 2004;33:612-7.

20 Brulhart MI, Wermeille JP. Multidisciplinary medication review: evaluation of a pharmaceutical care model for nursing homes. Int $J$ Clin Pharm 2011;33:549-57.

21 Spinewine A, Swine C, Dhillon S, et al. Effect of a collaborative approach on the quality of prescribing for geriatric inpatients: a randomized, controlled trial. J Am Geriatr Soc 2007;55:658-65.

22 Betegnie AL, Patin S, Debray M, et al. Optimisation de la prise en charge médicamenteuse du sujet âgé dans les établissements sanitaires et médicosociaux: une démarche qualité transversale de la filière gérontologique Annecy-Rumilly-Saint Julien-Gex-Bellegarde. Cah Année Gérontologique 2014;6:65-72.

23 Ouvrier M, Dobremez V, Debray M, et al. Relecture pluridisciplinaire d'ordonnances: retour d'expérience d'un hôpital à propos d'une optimisation concrète de la prise en charge médicamenteuse du sujet âgé. Cah Année Gérontologique 2014;6:73-8.

24 Deschodt M, Flamaing J, Haentjens P, et al. Impact of geriatric consultation teams on clinical outcome in acute hospitals: a systematic review and meta-analysis. BMC Med 2013;11:48.

25 Salles N. Enquête nationale sur les pratiques des Equipes Mobiles de Gériatrie en France. Groupe de travail des équipes mobiles de gériatrie de la SFGG. Rev Gériatrie 2012;31:738.

26 Dalleur O, Boland B, Losseau C, et al. Reduction of potentially inappropriate medications using the STOPP criteria in frail 
older inpatients: a randomised controlled study. Drugs Aging 2014;31:291-8.

27 Schmader KE, Hanlon JT, Pieper CF, et al. Effects of geriatric evaluation and management on adverse drug reactions and suboptimal prescribing in the frail elderly. Am J Med 2004;116:394-401.

28 Chan A-W, Tetzlaff JM, Altman DG, et al. Spirit 2013 statement: defining standard protocol items for clinical trials. Ann Intern Med 2013;158:200-7.

29 Eldridge SM, Chan CL, Campbell MJ, et al. Consort 2010 statement: extension to randomised pilot and feasibility trials. BMJ 2016;355:i5239.

30 Lemaire F, Marchenay B, Chassany O, et al. The European 'clinical trial' regulation: relationship with the Jardé act: a giens workshop. Therapies 2015;70:29-36.

31 Schoenmakers TWA, Wensing M, De Smet PAGM, et al. Patientreported common symptoms as an assessment of interventions in medication reviews: a randomised, controlled trial. Int J Clin Pharm 2018;40:126-34.
32 Allenet B, Juste M, Mouchoux C, et al. De la dispensation au plan pharmaceutique personnalisé: vers un modèle intégratif de pharmacie clinique [From dispensing to personal pharmaceutical plan: Towards an integrated model of clinical pharmacy]. Pharm Hosp Clin 2019;54:56-63.

33 Motter FR, Fritzen JS, Hilmer SN, et al. Potentially inappropriate medication in the elderly: a systematic review of validated explicit criteria. Eur J Clin Pharmacol 2018;74:679-700.

34 Hanlon JT, Schmader KE, Samsa GP, et al. A method for assessing drug therapy appropriateness. J Clin Epidemiol 1992;45:1045-51.

35 World Health Organisation (WHO). Falls, 2020. Available: https:// www.who.int/news-room/fact-sheets/detail/falls

36 Charlson ME, Pompei P, Ales KL, et al. A new method of classifying prognostic comorbidity in longitudinal studies: development and validation. J Chronic Dis 1987;40:373-83.

37 Trivalle C, Cartier T, Verny C, et al. Identifying and preventing adverse drug events in elderly hospitalised patients: a randomised trial of a program to reduce adverse drug effects. $J$ Nutr Health Aging 2010;14:57-61. 